\title{
Conception, réalisation et dévasage des ouvrages de prise et de rejet d'eau de refroidissement de la centralle du Blayais
}

\section{Desilting of intake and outlet works of the Blayais plant}

\author{
J. Laurent, M. Davesne, $\mathbb{F}$. Lemelle et J. Ricco \\ Electricité de France, Région d'Equipement «Paris»
}

\section{Généralités}

La Centrale électronucléaire du Blayais est installée en rive droite de la Gironde, à $50 \mathrm{~km}$ en aval de Bordeaux, au centre d'un marais de 6000 hectares (Fig. 1).

L'implantation de la Centrale sur ce site, correspond aux besoins énergétiques régionaux et en particulier à la possibilité d'utiliser le débit important, qui transite à chaque marée, pour assu rer, en circuit ouvert, le refroidissement de 4 tranches de $900 \mathrm{MW}$ de la filière PWR (Fig. 2).

Le débit d'eau de refroidissement principal est de $168 \mathrm{~m}^{3} / \mathrm{s}$ avec un échauffement au condenseur de $11,5^{\circ} \mathrm{C}$.

Après une description des ouvrages de prise et de rejet d'eau et de leur fonctionnement, cette communication montre comment la forte quantité de sédiments rencontrés et leur accumulation, qui s'écartait de ce qui était prévu, ont conduit à modifier en partie l'ouvrage initial et à prendre des précautions originales lors des mises en service.

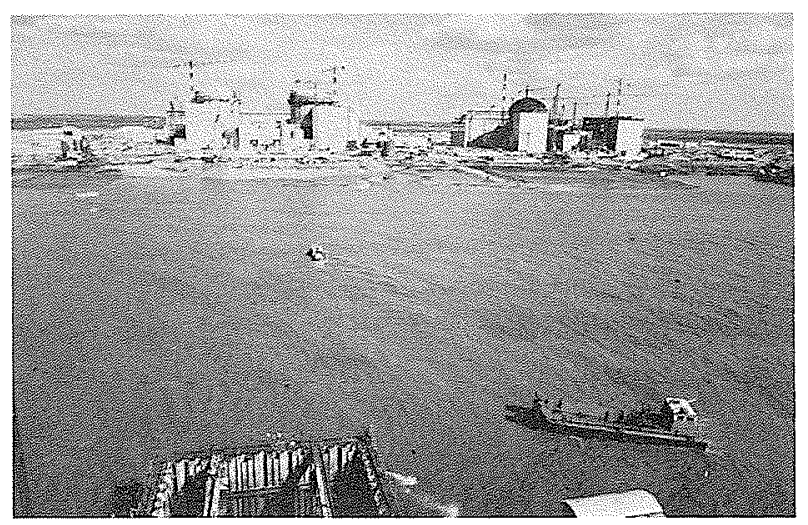

Figure 1 - Vue générale du site.

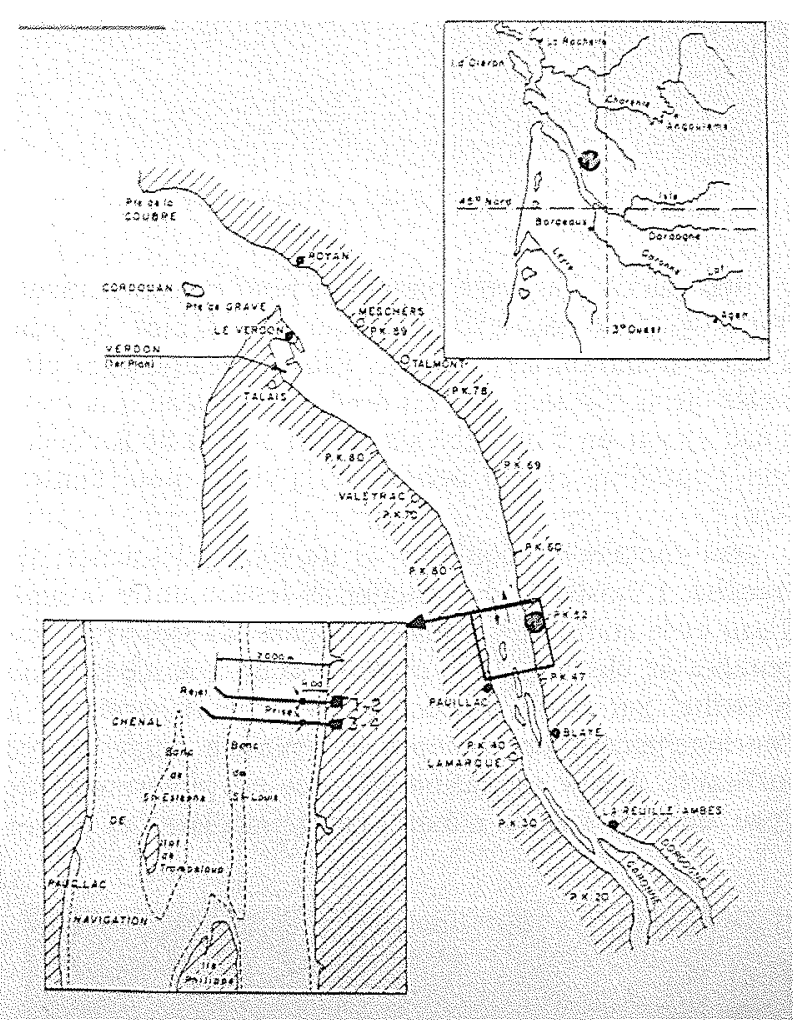

Figure 2 - Situation générale du site sur l'estuaire.

\section{Description et fonctionnement}

\subsection{La Prise d'eau :}

Elle est constituée par (Fig. 3):

- une prise d'eau proprement dite commune à deux tranches de $900 \mathrm{MW}$, située au dessous du niveau des plus basses eaux, à $400 \mathrm{~m}$ environ de la rive. 


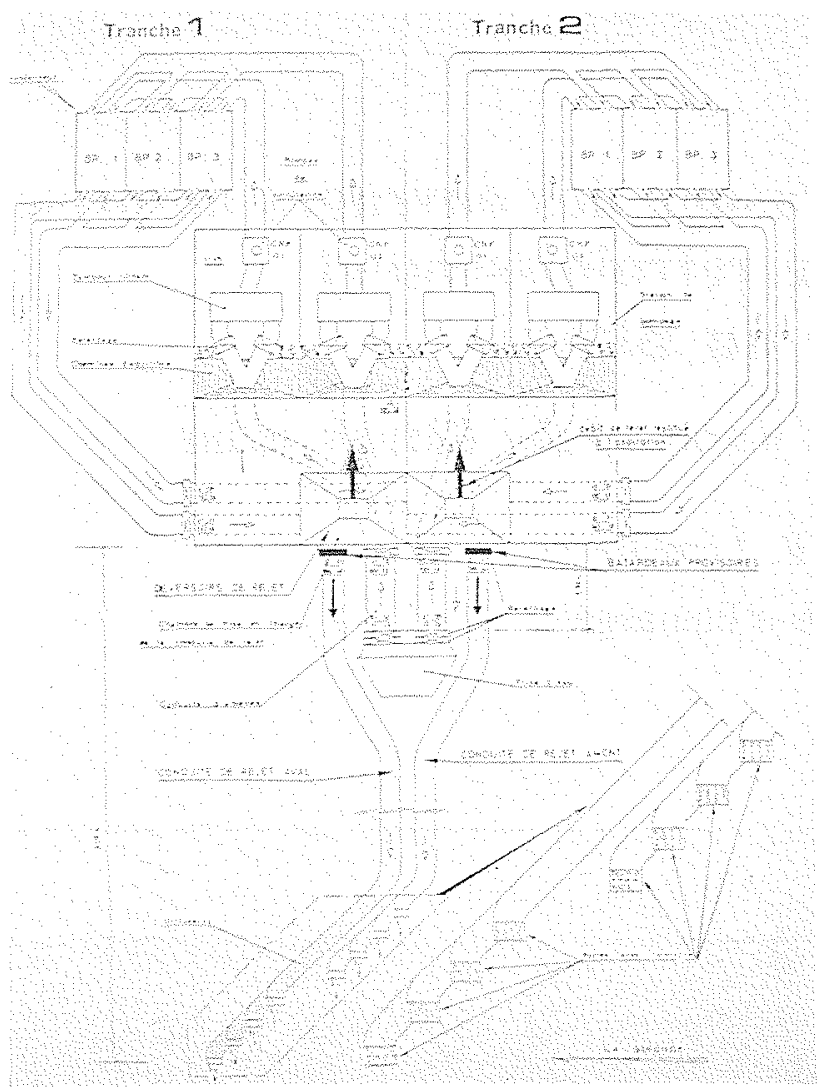

Figure 3 - Ouvrages d'eau - Plan schématique.

- une galerie d'amenée principale pour chaque tranche, d'un diamètre de $4,70 \mathrm{~m}$, ensouillée, reliant la prise d'eau à la station de pompage. Chaque galerie peut être isolée de la Gironde par un double batardeau à la prise d'eau et par un batardeau simple en amont de la cheminée d'équilibre ce qui permet de la visiter pour l'entretien.

- une cheminée d'équilibre et un bassin d'amenée par tranche. L'existence des galeries de liaison entre la prise d'eau et la station de pompage impose, à l'aspiration des pompes de circulation, la présence d'un volume tampon constitué par la cheminée d'équilibre pour permettre à l'enclenchement des pompes le maintien d'une charge suffisante à leur aspiration (la mise en mouvement de l'eau dans la galerie n'étant pas immédiate) et à leur

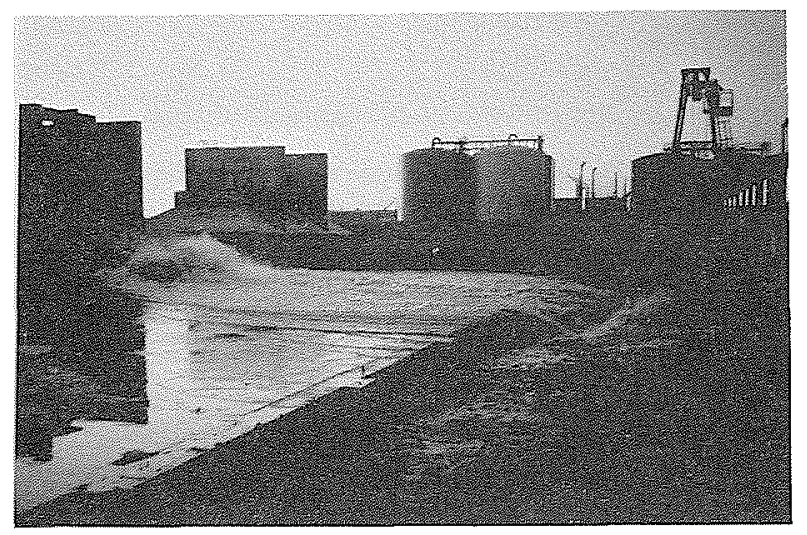

Figure 4 - Déversoir - Déversement par débordement. déclenchement, d'éviter le débordement au droit des tambours filtrants en particulier, l'arrêt de la masse d'eau en mouvement dans les galeries n'étant pas non plus instantané.

- deux pompes de circulation et quatre pompes d'eau brute secourues par tranche prélevant l'eau dans la cheminée d'équilibre au travers de deux tambours filtrants.

Il est impossible de démarrer une pompe de circulation si le niveau dans la cheminée d'équilibre est inférieur à une valeur minimale, et on peut être amené à différer ce démarrage jusqu'à ce que les oscillations dues aux perturbations soient amorties et que le niveau en Gironde soit suffisant.

\subsection{Le Rejet d'eau :}

Une disposition particulière des ouvrages de rejet a été adoptée, afin d'améliorer la dilution thermique et d'éviter l'envasement.

Pour ces raisons, les ouvrages de rejet sont communs à deux tranches. lls sont constitués de :

- deux déversoirs, chacun d'cux (Fig. 3) recevant les eaux provenant d'un demi-condenseur de chacune des tranches et déversant celles-ci dans une galerie de rejet. En régime transitoire, il y a également possibilité de déversement par débordement du déversoir dans le bassin d'amenée, puis dans la cheminée d'équilibre, et recirculation d'une partie de l'eau de circulation (Fig. 4). - deux galeries de rejet, d'un diamètre de $4,30 \mathrm{~m}$, ensouillées, reliant chacun des déversoirs aux ouvrages de rejet d'eau et isolables en aval des déversoirs.

Du fait de la particularité signalée ci-dessus, si une des deux tranches est à l'arrêt, chacun des deux déversoirs reçoit le débit d'une pompe de la tranche voisine. Chaque conduite de rejet est donc parcourue par la moitié de son débit nominal.

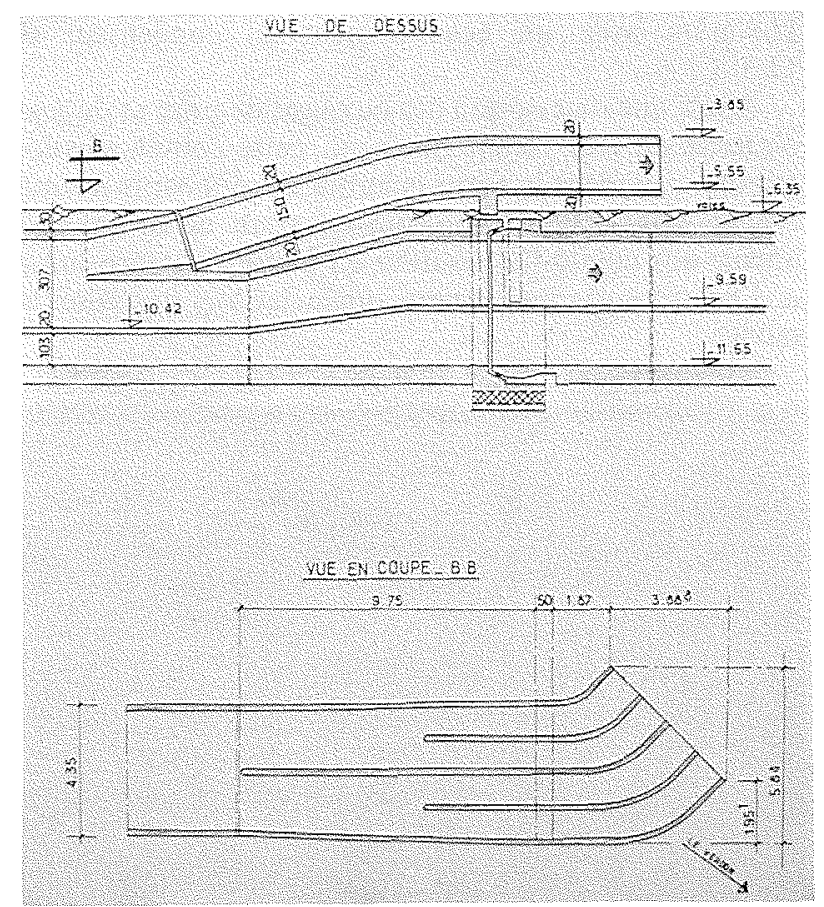

Figure 5 - Schéma d'un diffuseur. 


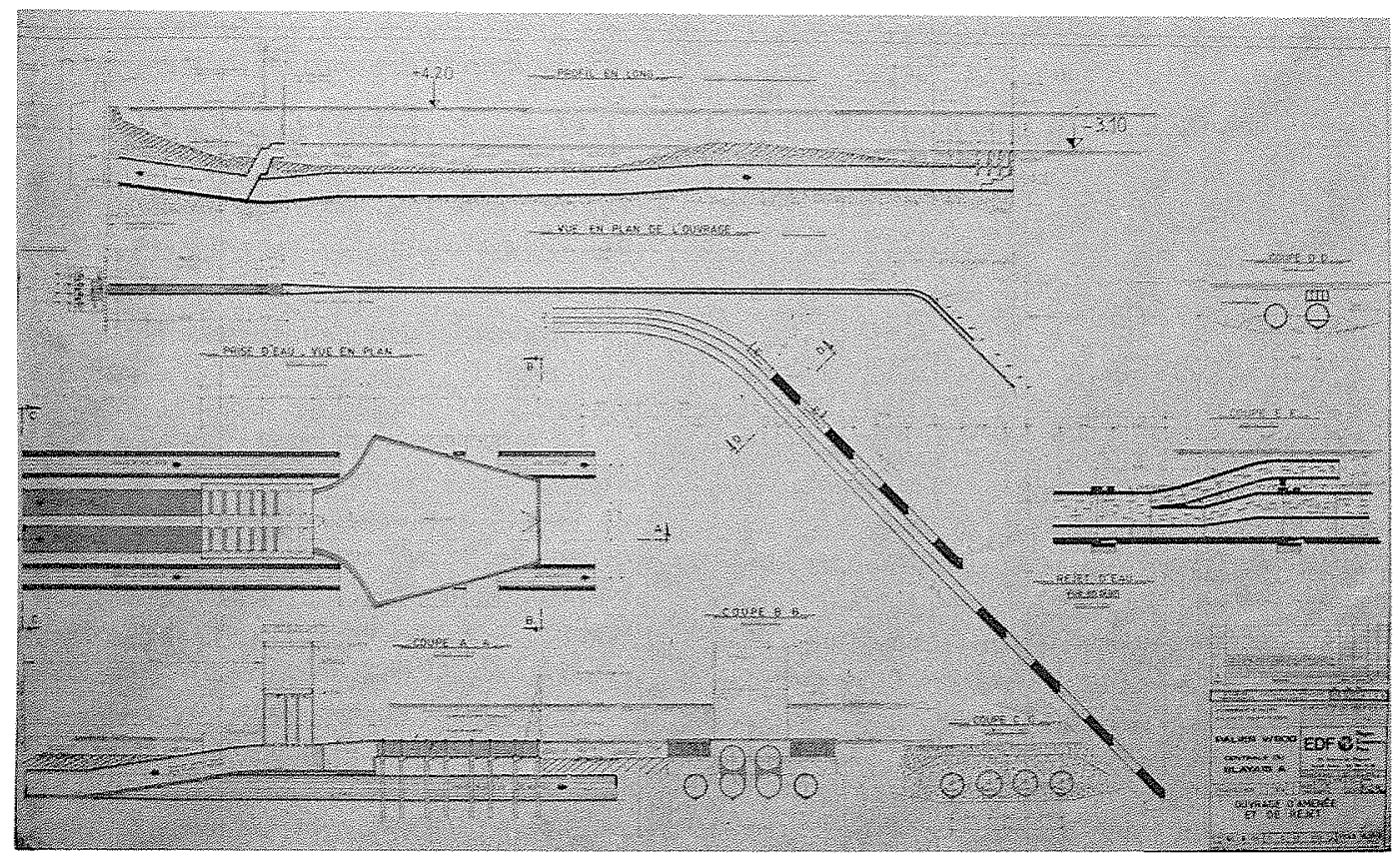

Figure 6 - Plan général de réalisation.

- deux ouvrages de rejet d'eau, constitués chacun de 4 diffuseurs situés dans le chenal médian de l'estuaire, à $2000 \mathrm{~m}$ de la rive (Fig. 5).

Par ailleurs chaque ensemble "tambour filtrant, pompe, demi-condenseur" est isolable par des batardeaux pour en permettre l'entretien.

\section{Réalisa tion des ouvrages (Fig. 6)}

La réalisation des ouvrages a donné lieu à d'importants travaux aussi bien sur terre que sur mer. On distingue les campagnes principales suivantes :
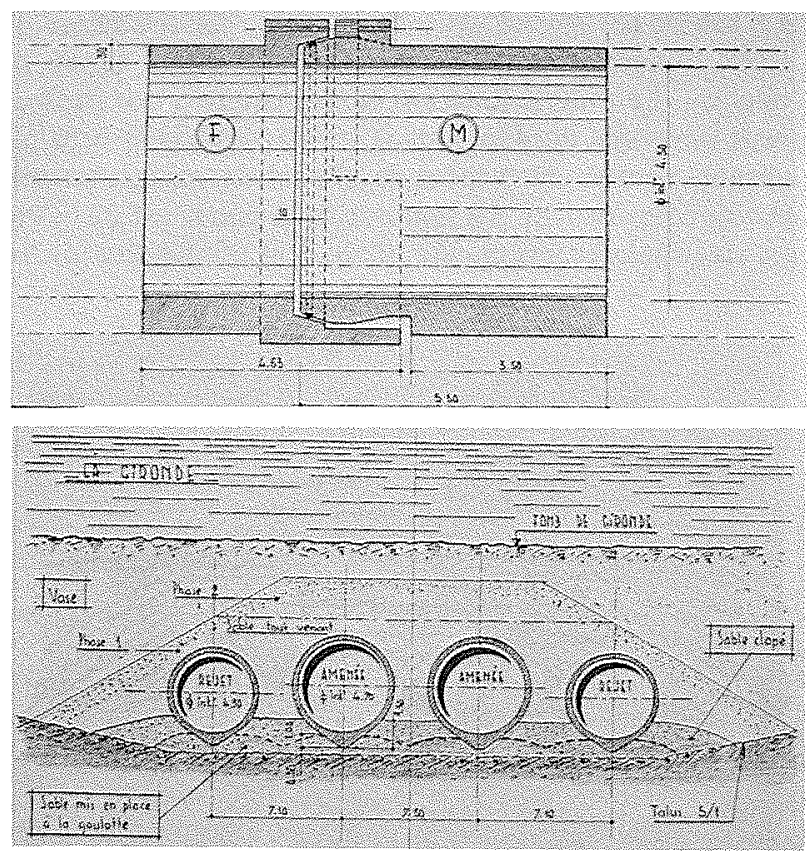

Figure 7 - Conduite - Détail d'un raccordement - Coupe.
- construction et pose des tuyauteries d'amenée et de rejet ;

- réalisation des prises d'eau en mer ;

- exécution des ouvrages terrestres, principalement la station de pompage ;

- raccordement des tuyauteries à la station de pompage.

\subsection{Pose des tuyauteries :}

Les tuyauteries préfabriquées, par éléments de $5 \mathrm{~m}$ en béton armé assemblés par des câbles de précontrainte pour former des caissons de $25 \mathrm{~m}$ pesant $400 \mathrm{t}$ environ, et obturés à chacune de leurs extrémités, furent amenés par flottaison sur le lieu de pose où une souille avait été préalablement réalisée par dragage (Fig. 7).

Les courants importants en Gironde, pouvant aller jusqu'à 6 noeuds avec des marées à haut coefficient, et la nature des eaux très chargées en limon ne permettent pas l'immersion de conduites avec des moyens traditionnels. Il a fallu construire un bateau spécial pour placer chaque élément de tuyau dans la souille creusée au fond du lit de la Gironde (catamaran constitué par deux moitiés de chaland métallique reliées par une superstructure supportant des treuils de pose) et mettre au point des systèmes de positionnement horizontaux et verticaux. (L'alignement est obtenu par des faisceaux Laser installés à terre et repris sur écran sur le bateau. La position en distance sur cette direction découle d'une triangulation par ondes courtes à partir de répondeurs à terre distants de $1900 \mathrm{~m}$ environ. Le calage définitif du bateau fut mesuré par instrument optique sur une borne fixe à terre. Les mesures de positionnement vertical furent faites à l'écho-sondeur doublées par des sondages à la chaîne. L'ensemble de l'opération fut contrôlé par des plongeurs sous-marins).

Après enlèvement des obturateurs d'extrémités, la tuyauterie était descendue et son extrémité mâle 


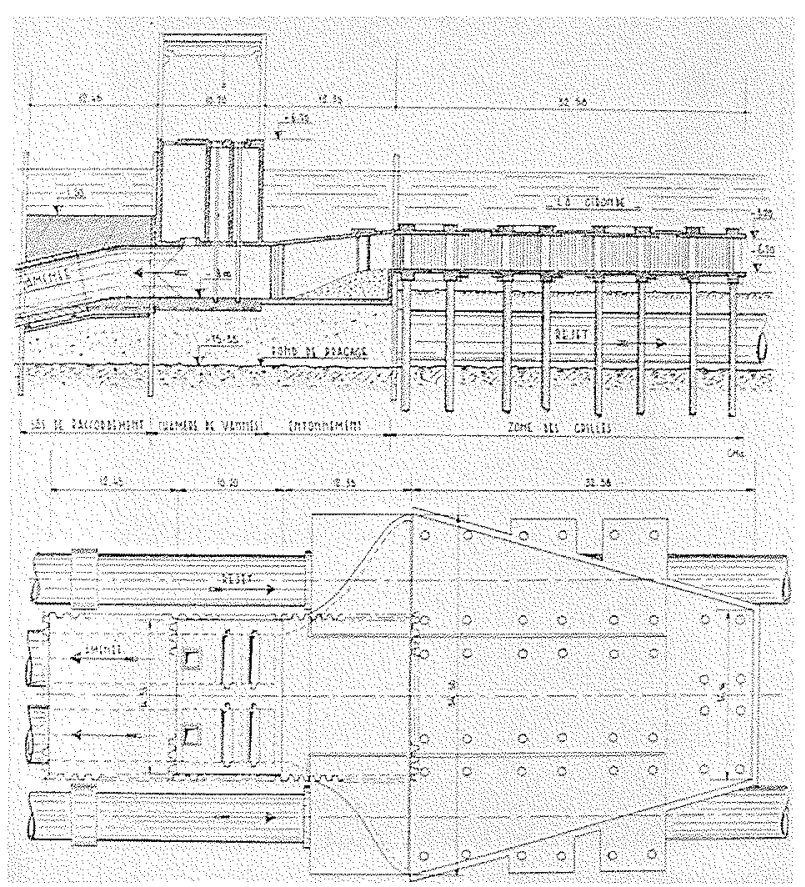

Figure 8 - Prise d'eau - Vue en plan - Coupe.

emboittée avec l'extrémité femelle de la tuyauterie déjà en place. Un bourrage de sable venait ensuite caler les tuyauteries qui étaient définitivement bloquées, après un avancement suffisant de pose, par un clapage de matériaux qui reconstituait le lit de la Gironde.

Le problème principal rencontré a été un envasement de la souille beaucoup plus rapide et plus important que prévu et dû vraisemblablement au brassage produit par les engins. Cet envasement avait été estimé à $0,50 \mathrm{~m}$ d'épaisseur par jour et il a été constaté suivant les zones de travail (chenal de Saintonge - Banc de StLouis) des épaisseurs de $1,20 \mathrm{~m}$ à $2 \mathrm{~m}$. L'enlèvement de cette vase a été réalisé par pompage depuis le catamaran de pose. La conséquence en a été le doublement des cadences de pose.

\subsection{Réalisation des prises d'eau :}

Les prises d'eau comprennent deux parties, la "tête de vipère" et la chambre des vannes.

La tête de vipère, qui doit son nom à sa forme, comporte $200 \mathrm{~m}^{2}$ de grilles latérales intégrées dans des éléments préfabriqués; ces éléments furent immergés à la grue sur des pieux battus (Fig. 8).

La chambre des vannes destinée au stockage des batardeaux a été réalisée de deux façons différentes:

- pour la prise d'eau des tranches 1 et 2 , la construction s'est effectuée de façon traditionnelle dans une enceinte de palplanches mais avec beaucoup de difficultés dues à la présence d'un fond karstique favorisant des venues d'eau importantes et à la pollution par les sédiments des bétons immergés.

- pour la prise d'eau des tranches 3 et 4 , la construction s'est effectuée, comme pour la tête de vipère, par éléments préfabriqués disposés sur des pieux. La principale difficulté fut d'atteindre une précision suffisante dans le positionnement relatif des pieux.

\subsection{Exécution des stations de pompage :}

Comme l'ensemble du bloc usine, les stations de pompage (Fig. 9) furent exécutées à l'intérieur d'un énorme caisson constitué par une paroi moulée dans le sol étanché au fond par un rabattement de nappe.

\subsection{Raccordement des tuyauteries à la station de pom- page:}

Le raccordement des tuyaux avec la station de pompage à travers la paroi moulée qui ceinture les fondations de l'usine a nécessité d'importants travaux : il a fallu construire un sas séparé de la Gironde par un véritable barrage de terre et de béton, et dans ce sas asséché, réaliser le raccordement après percement de la paroi.

Afin d'éliminer la possibilité de déboitement des tuyauteries en charge au sein du barrage par déplacement de la digue à la vidange, il a été nécessaire de s'assurer de sa stabilité en laissant subsister le minimum de vase en place au terrassement et éviter qu'au cours des remblais la vase ne vienne se déposer. Un dévasage préalable à la constitution de la digue et la cadence des remblais sont fondamentaux.

\section{Conception initiale}

La structure des ouvrages de rejet a été définie à partir d'études effectuées essentiellement sur modèles réduits afin, d'une part, d'éviter la recirculation avec la prise d'eau et, d'autre part, d'assurer la dilution initiale des calories, la plus forte possible, tout en minimisant l'impact hydraulique et sédimentologique des ouvrages.

\subsection{Caractéristiques de l'estuaire au niveau du site - Importance des sédiments}

Au niveau de la centrale, l'estuaire a une largeur de $4,5 \mathrm{~km}$ et est séparé en trois chenaux par des bancs de sable longitudinaux émergeants à la marée basse de vive-eau. Le chenal longeant la rive droite a une profondeur moyenne de $4 \mathrm{~m}$ sous le 0 des plus basses eaux, le chenal médian de $3 \mathrm{~m}$ et le chenal de navigation une profondeur de $7 \mathrm{~m}$. La hauteur de la marée est de $5,3 \mathrm{~m}$ en vive-eau exceptionnelle et de $3 \mathrm{~m}$ en morte-eau. Au

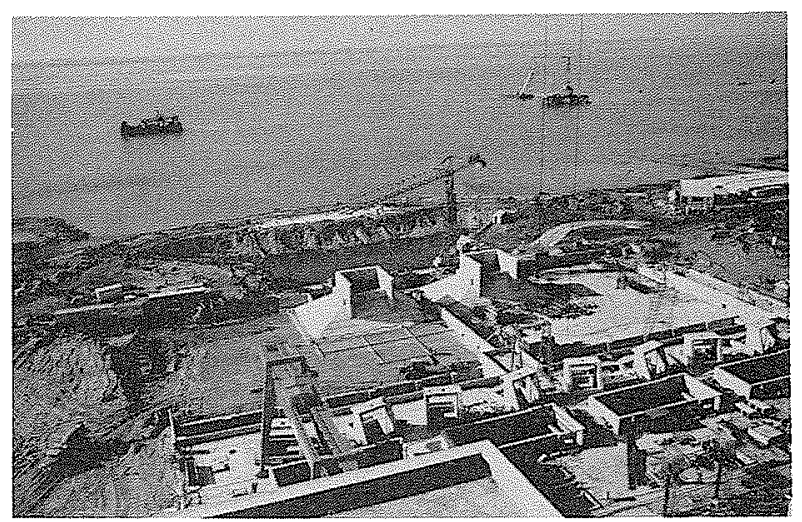

Figure 9 - Station de pompage en construction. 
cours de la marée, la vitesse moyenne sur la verticale dans le chenal médian atteint un maximum de $1,50 \mathrm{~m} / \mathrm{s}$ en vive-eau et de $1,00 \mathrm{~m} / \mathrm{s}$ en morte-eau.

En étiage, la salinité au cours de la marée varie entre $5 \%$ à basse-mer et $10 \%_{0}$ à pleine-mer. En forte crue, la salinité est pratiquement nulle. En raison de l'intensité des courants, le gradient vertical de salinité est faible même en morte-eau; il en est de même du gradient vertical de température.

Les bancs de sable longitudinaux, en particulier le banc de Saint-Estèphe, jouent un rôle important dans le guidage des courants de jusant et par suite pour le maintien des profondeurs du chenal de navigation en rive gauche. Les ouvrages d'eau de la centrale ne doivent donc pas compromettre leur stabilité.

Dans un estuaire tel que la Gironde, les eaux douces provenant des fleuves et charriant des sédiments rencontrent les eaux marines introduites au gré des marées. Il en résulte des phénomènes complexes de circulation qui concourent à la formation d'un "bouchon vaseux", zone à concentration relativement élevée de sédiments en suspension.

Les dimensions et l'emplacement du bouchon vaseux varient de façon périodique à l'échelle de la marée, à l'échelle du cycle de marée, et surtout à l'échelle des saisons (en fonction des débits fluviaux).

$\mathrm{Au}$ phénomène de formation du bouchon vaseux se rattache le phénomène de formation de la "crême de vase", constituée de lentilles très turbides (100 à 300 g/1) qui se décantent dans les dépressions situées au fond des chenaux, à condition que la profondeur soit suffisante.

Pour des sédiments tels que ceux rencontrés en Gironde, la vitesse de circulation en dessous de laquelle commence la décantation est d'environ $0,5 \mathrm{~m} / \mathrm{s}$; la vitesse de remise en suspension est de $0,5 \mathrm{~m} / \mathrm{s}$ à $1,5 \mathrm{~m} / \mathrm{s}$.

\subsection{Prise d'eau}

\subsubsection{Définition des prises d'eau}

Les ouvrages des tranches 3 et 4 sont situés à $350 \mathrm{~m}$ en amont de ceux des tranches 1-2 (Fig. 2). Les prises d'eau de chaque paire de tranches sont situées dans le chenal rive droite à $400 \mathrm{~m}$ de la berge afin de s'éloigner suffisamment des zones peu profondes fréquentées par des organismes marins migrateurs et pour disposer de profondeurs d'eau suffisantes afin que l'ouvrage reste constamment immergé.

La forme de la prise a été étudiée pour obtenir une vitesse de prélèvement aussi homogène que possible et inférieure à $0,40 \mathrm{~m} / \mathrm{s}$ perpendiculairement au courant dans le souci d'éviter au maximum l'aspiration d'alevins et de poissons. En revanche, le convergent qui amène le débit aux deux conduites est conçu pour que les vitesses soient supérieures à $0,80 \mathrm{~m} / \mathrm{s}$ en fonctionnement normal pour éviter la sédimentation des vases.

\subsubsection{Définition des conduites:}

Les conduites sont complètement ensoullées pour ne pas créer un obstacle transversal dans l'estuaire. Ces conduites d'amenée, pour répondre à des critères de sûreté, sont visitables; cette particularité nécessite un chargement par un lest et un batardage aux extrémités.
La section des conduites a été déterminée pour qu'à la moitié du débit nominal la vitesse obtenue soit telle que les sédiments ne se déposent pas. Vérification a été faite de la compatibilité des pertes de charges engendrées avec les hauteurs manométriques des pompes.

\subsection{Rejet}

\subsubsection{Position des ouvrages - prise en compte des effets de recirculation}

L'étude préliminaire de l'impact thermique sur un modèle à fonds fixes, au $1 / 2000^{\mathrm{e}}$ en plan et $1 / 200^{\mathrm{e}}$ en hauteur, représentant l'ensemble de l'estuaire, a montré qu'un rejet situé également en rive droite conduisait à une forte recirculation, le panache d'eau chaude suivant la berge et se mélangeant mal transversalement entre l'instant de rejet et les renverses de courant.

Il a donc été décidé d'utiliser la séparation naturelle du chenal médian et du chenal rive droite due au banc de Saint-Louis en implantant le rejet dans le chenal médian à $2 \mathrm{~km}$ environ de la rive, les ouvrages étant reliés à la centrale par des conduites ensouillées. Dans ces conditions, la recirculation à la prise est en moyenne inférieure à $0,5^{\circ} \mathrm{C}$ dans les conditions défavorables d'un débit fluvial d'étiage et d'un fonctionnement continu de la centrale à pleine puissance.

\subsubsection{Amélioration de la dilution - Définition des diffu- seurs}

A partir d'études effectuées sur modèles réduits, la recherche de la dilution initiale des calories la plus forte possible, compatible avec la présence de fonds sableux et vaseux d'un chenal de navigation et de faibles profondeurs d'eau à marée basse, a conduit à un fractionnement du débit total rejeté de $168 \mathrm{~m}^{3} / \mathrm{s}$ en 16 débits unitaires de $10,5 \mathrm{~m}^{3} / \mathrm{s}$. Le diffuseur des tranches 1 et 2 est constitué de huit buses de rejet écartées de $28,5 \mathrm{~m}$ et rejetant perpendiculairement au courant moyen en Gironde. Le diffuseur des tranches 3 et 4 est identique mais il est situé $350 \mathrm{~m}$ plus à l'amont et décalé vers le large par rapport à celui des tranches 1 et 2 afin d'éviter l'interférence des différents panaches.

\subsubsection{Vérification de l'impact courantologique et sédi- mentologique}

Cette étude a été effectuée sur un modèle physique représentant la partie centrale de l'estuaire. Ce modèle, construit dans le cadre d'une étude d'aménagement portuaire pour le compte du Port Autonome de Bordeaux, a permis de vérifier que les bancs de sable restaient stables en présence des rejets de la centrale et que les érosions occasionnées par les jets étaient limitées au voisinage immédiat des ouvrages. Ces érosions sont évitées par une protection locale des fonds autour des ouvrages.

\section{Evolution du projet et précautions prises contre l'envasement}

Si les principales options de conception de la station de pompage et des ouvrages de prise et de rejet d'eau 
étaient arrêtées assez tôt, un certain nombre de modifications devaient être envisagées, alors que les travaux étaient déjà avancés.

Ces aménagements, qui étaient destinés à satisfaire des impératifs d'exploitation, ont permis de faire face à certaines difficultés de réalisation, non mises en évidence au stade du projet.

\subsection{Modification de la station de pompage}

Au stade de l'avant-projet, seule la construction du bassin d'amenée était envisagée. Pour éviter l'envasement de ce bassin, des moyens de brassage importants étaient nécessaires (racleurs oscillants, éjecteurs à air comprimé ou pont racleur étaient étudiés).

Cet avant-projet fut rejeté du fait des contraintes qu'entra inait l'exploitation de telles installations, et il fut décidé de construire en amont des tambours filtrants, une cheminée d'équilibre dont le profil des fonds ne permettait pas l'accrochage des vases. Le bassin d'amenée était également surélevé de manière à n'être mis en eau que sur les transitoires d'exploitation, ou lors des hautes eaux d'une marée de fort coefficient.

\subsection{Prise en compte des effets de marnage}

Lorsqu'une tranche est à l'arrêt, le volume constitué par la cheminée d'équilibre et l'alvéole des filtres est soumis au marnage, ce qui entraîne le renouvellement périodique de l'eau à l'intérieur de la galerie d'amenée.

En prenant l'hypothèse d'une eau chargée de $5 \mathrm{~g} / 1$ de matière en suspension, on dépose 16,5 tonnes de vase par marée. Compte tenu des caractéristiques de ces boues on peut estimer, dans l'hypothèse d'un envasement uniforme sur les 200 premiers mètres de la galerie, que la hauteur de dépôt sera de $1,4 \mathrm{~m}$ en 8 jours, $2 \mathrm{~m}$ en 15 jours et $4 \mathrm{~m}$ au bout de 2 mois.

Les galeries de rejet sont également soumises par le déversoir à l'influence du marnage. On peut estimer que 6 à 7 tonnes de vase se déposent par marée, lorsqu'il n'est établi aucune circulation dans la galerie. La hauteur de vase sur les 100 derniers mètres de la galerie sera de $1,3 \mathrm{~m}$ après 8 jours d'arrêt et de $3,6 \mathrm{~m}$ au bout de 4 mois.

Comme on le voit, les ouvrages étant susceptibles de s'envaser pendant les périodes d'arrêt, on fut donc amené à construire des batardeaux sur les galeries d'amenée et de rejet, au droit de la station de pompage, de manière à pouvoir intervenir dans des délais restreints et limiter l'envasement.

\subsection{Envasement par le circuit d'eau brute secourue}

Dans le cas d'indisponibilité des 4 pompes de circulation d'une paire de tranches, les galeries d'amenée sont traversées par le seul débit des circuits d'eau brute secourus. La vitesse de l'eau y est alors de quelques centimètres par seconde, et les matières en suspension décantent et envasent progressivement les galeries. L'envasement se poursuit jusqu'à ce que la section non obturée ae la galerie soit telle que la vitesse de l'eau ne permette plus la décantation $(\# 0,5 \mathrm{~m} / \mathrm{s})$. On obtient alors une section d'équilibre qui permet d'assurer l'alimentation du circuit d'eau brute secouru (circuit SEC).
Par ailleurs, une règle de sûreté impose une seconde file indépendante capable, depuis la source froide, d'assurer la fonction de refroidissement. Pour satisfaire à cette règle il fut réalisé la possibilité de mettre en communication les cheminées d'équilibre d'une même paire de tranches ce qui permet, en cas de nécessité, d'alimenter les circuits SEC par l'une ou l'autre des galeries d'amenée principales.

La mise en communication des cheminées d'équilibre peut également être utilisée pour effectuer le nettoyage d'une galerie d'amenée envasée comme on le verra plus loin.

\subsection{Mise au point de procédure de dévasage}

Les possibilités d'envasement étant admises, il ne peut être question de démarrer une pompe de circulation de la tranche dont la galerie est envasée (la perte de charge dans la conduite étant trop importante, on viderait la cheminée d'équilibre au moment du démarrage). On est donc conduit à nettoyer la galerie.

\section{Première mise en service - Dévasage}

Les phénomènes de tassement et de consolidation de la vase sont importants et ont été étudiés par différents organismes (Laboratoire Central d'Hydraulique de France, Institut Géologique du Bassin Aquitaine, LNH), mais rien n'a été étudié au-delà d'un délai de 7 mois et, le plus souvent, les essais ont été faits avec de l'eau douce. A la mise en service des ouvrages, la vase avait séjourné deux ans dans les tuyauteries posées en premier.

Au point de vue densité et cohésion de la vase, il semble y avoir un écart entre les résultats de laboratoire et les observations "in situ", ces dernières étant plus pessimistes. Dans le but de lever l'incertitude sur les comportements de la vase à l'intérieur des tuyauteries, il fut décidé d'effectuer des prélèvements qui montrèrent que des dépôts s'étaient formés sur 3,50 m de haut avec une concentration variant de $800 \mathrm{~g} / 1$ près $\mathrm{du}$ fond (vase consolidée) à $450 \mathrm{~g} / \mathrm{l}$ en surface ; la granulométrie de cette vase était fine, la quasi-totalité des grains étant au-dessous de 60 micromètres.

Une telle rapidité du dépôt ainsi que les concentrations atteintes, qui ont dépassé toutes les prévisions, ont plusieurs causes, en particulier :

- au moment de la pose des conduites, il y a un important brassage de boue (densité de la vase 100 à $150 \mathrm{~g} / \mathrm{l}$ ) et le phénomène de décantation est important $(1,50$ à $1,60 \mathrm{~m}$ ),

- des courants dûs aux variations de densité apparaissent, mais sont difficilement quantifiables.

- les obturateurs d'extrémité n'étant pas complètement étanches, on peut penser que la vase s'introduit dans les conduites par diffusion, puis est transportée le long de ces conduites par de faibles courants de convection favorisés par le mamage. Cependant leur influence ne semble pas intéresser une longueur de tuyauterie supérieure à $200 \mathrm{~m}$ si une des extrémités de la conduite n'est pas fermée hermétiquement.

En tout état de cause, des moyens particuliers durent être mis en oeuvre. 


\subsection{Dévasage des conduites d'amenée}

Les conduites d'amenée ne posaient pas de problème pour le dévasage. En effet, ces conduites peuvent être isolées de la Gironde grâce aux batardeaux prévus à la prise d'eau. Elles sont alors accessibles après avoir été vidangées. Le dévasage a donc pu être réalisé après achèvement des ouvrages et assèchement des conduites. Il a consisté à attaquer le front de vase avec une lance à gros débit qui remettait la vase en suspension.

L'eau chargée de vase était pompée au fur et à mesure et rejetée dans la Gironde.

\subsection{Dévasage des conduites de rejet}

Les conduites de rejet, longues de $2000 \mathrm{~m}$, sont inacessibles aux plongeurs et ne peuvent être dévasées par des moyens mécaniques connus. La solution adoptée à consisté à éroder la vase par le passage du débit des pompes de circulation.

L'étude du programme de dévasage a été effectuée à l'aide d'un modèle mathématique traité par ordinateur.

\subsubsection{Principe}

Afin de se prémunir contre des risques de colmatage en particulier aux diffuseurs de rejet sous l'effet d'un dévasage trop brutal, et compte tenu de l'impossibilité de régler le débit fourni par les pompes, le débit de circulation dans les conduites fut augmenté progressivement en mettant en service une pompe puis deux pompes, en utilisant la variation de la charge motrice avec la marée et en ouvrant par étapes un batardeau provisoire situé à l'entrée des conduites après le déversoir de rejet (Fig. 3), le débit des pompes ne passant pas dans la conduite étant évacué par le déversoir de rejet.

Le principe des opérations de dévasage a été le suivant (Fig. 10) :

- ouverture à une cote donnée du batardeau constitué d'éléments amovibles,

- démarrage à marée haute d'une ou de deux pompes de circulation. Le débit passant dans la conduite de rejet augmente lorsque la chambre de mise en charge se remplit puis se stabilise à une valeur fonction de la perte de charge singulière du batardeau et de la perte de charge dans la conduite envasée; le débit ne passant pas dans la conduite de rejet est évacué par le déversoir de rejet et est recyclé à l'aspiration des pompes,

- à marée descendante, la charge motrice (différence entre la hauteur d'eau dans le déversoir de rejet et la cote en Gironde) et par suite le débit et la vitesse de circulation augmentent. Sous l'effet de l'augmentation régulière de vitesse, le dépôt de vase s'érode progressivement ;

- à basse-mer, la section de passage atteint un état d'équilibre tel que la vitesse de l'eau soit égale à la vitesse de début d'érosion de la vase. La vitesse de début d'érosion crô̂t avec la concentration du dépôt de vase et par suite augmente au fur et à mesure de l'érosion du dépôt ; - arrêt des pompes et ouverture du batardeau à une nouvelle cote telle qu'à haute mer le debit soit voisin du débit atteint à la fin de l'étape précédente ;

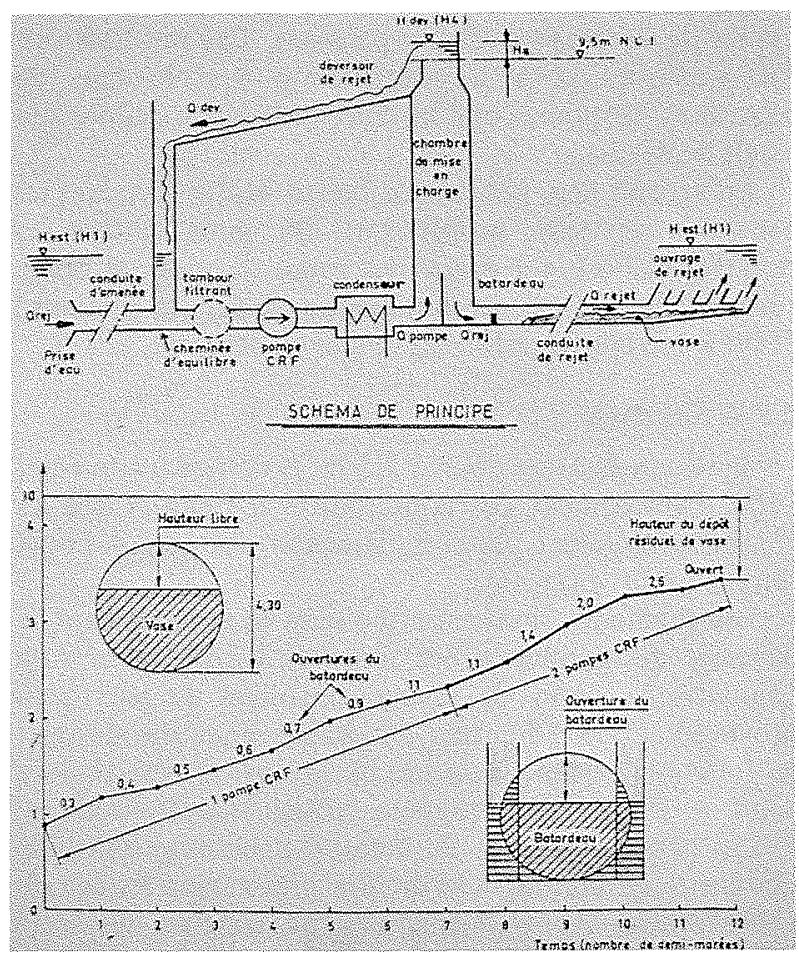

Figure 10 - Dévasage d'une conduite - Schéma de principe Prévision du dévasage d'une conduite obtenue à partir des résultats des calculs.

- démarrage des pompes à marée haute et érosion de la vase pendant la marée descendante, etc. .

$\mathrm{Au}$ cours de ces opérations, les mesures suivantes ont été effectuées:

- niveau d'eau dans la chambre de mise en charge. Cette mesure permet de connaître le débit sur le déversoir de rejet et par différence avec le débit de la pompe, le débit passant dans la conduite de rejet;

- niveau piézométrique à l'entrée de la conduite, à l'aval du batardeau qui donne la perte de charge due au batardeau;

- niveau d'eau en Gironde devant les diffuseurs de rejet. La différence entre ces deux derniers niveaux donne, après déduction des pertes de charge singulières dans les diffuseurs de rejet (qu'il n'est pas possible de mesurer) la perte de charge linéaire dans la conduite.

La connaissance du débit de passage et de la perte de charge linéaire permet d'en déduire la section libre dans la conduite et de suivre le dévasage progressif de la conduite.

\subsubsection{Etude théorique préalable}

Des calculs du débit d'eau dans la conduite en régime permanent en fonction des différents paramètres : ouverture du batardeau, niveau de la Gironde, hauteur de vase dans la conduite de rejet furent préalablement effectués.

A partir de la donnée de la répartition de la concentration entre la surface et le fond du dépôt de vase supposé de hauteur uniforme le long de la conduite et d'une loi de vitesse de début d'érosion en fonction de cette concentration, les résultats des calculs donnent les ouvertures du batardeau nécessaires pour assurer le pro- 
cessus de dévasage décrit auparavant. Ces résultats ont été appliqués pour la construction des éléments du batardeau et pour l'établissement de la procédure des essais de dévasage.

En second lieu, ces calculs furent utilisés pendant les essais de dévasage proprement dits pour obtenir les données élaborées à partir des essais de mesures effectuées (débit déversé, section de passage dans la conduite) et pour orienter et suivre les étapes de dévasage.

\subsubsection{Résultats}

Aprés un essai de débit à $2,5 \mathrm{~m}^{3} / \mathrm{s}$ destiné à apprécier l'état d'envasement des conduites et à s'assurer que les conduites n'étaient pas complètement obturées, les opérations furent engagées.

Les premières mesures effectuées in situ pendant le dévasage des conduites des tranches 1-2 ont permis de connaitre la perte de charge singulière du batardeau provisoire placé à l'entrée de chaque conduite de rejet en fonction de son ouverture et de suivre l'évolution du débit et de la section moyenne envasée dans la conduite.

Les pertes de charge du batardeau mesurées sont sensiblement différentes des valeurs prises dans les calculs prévisionnels. Après correction, on constate un assez bon accord entre l'évolution du débit et de la section envasée, observée et prédite, sauf à la fin de l'opération de dévasage.

En effet, le dévasage de la conduite a été complet en nature alors que l'on prévoyait un dépôt résiduel de $0,85 \mathrm{~m}$ environ. Cette différence provient vraisemblablement d'une répartition réelle de la concentration sur la hauteur du dépôt différente de celle prise par hypothèse.

Enfin, bien que l'on ne connaisse pas avec précision cette répartition et que les sections envasées soient déduites des mesures de perte de charge et non mesu. rées directement, les résultats de mesures semblent confirmer la loi de vitesse critique d'érosion d'un dépôt de vase de concentration supérieure à $450 \mathrm{~g} / 1$ déduite des résultats obtenus en laboratoire.

\subsection{Dévasage des diffuseurs}

Pour les tranches 1 et 2 avant le début du dévasage par les pompes de circulation, les diffuseurs amont de chacune des deux conduites avaient été dévasés, au prix d'assez grandes difficultés, par des plongeurs pénétrant dans l'ouvrage et utilisant des moyens mécaniques. Les trois autres diffuseurs de chaque conduite étaient presque complètement obstrués.

Comme on l'espérait, le deuxième et le troisième diffuseur de chaque conduite se sont dévasés d'euxmêmes, au fur et à mesure de l'augmentation du débit dans les conduites, mais les diffuseurs aval sont restés complètement bouchés. Ces derniers auraient pu être dévasés sur leur longueur $(20 \mathrm{~m})$ par les mêmes moyens que les diffuseurs amont, mais il n'était pas possible d'y envoyer les plongeurs faute de place.

Un autre moyen, évitant de pénétrer dans l'ouvrage a donc dû être employé. Il a consisté à utiliser un "hydrocureur" (engin en forme d'obus alimenté en eau sous très forte pression par un tuyau souple et envoyant vers l'arrière des jets d'eau qui le propulsent par réaction. En même temps, d'autres jets dirigés vers l'avant et latéralement désagrègent la vase).

Cet engin, introduit plusieurs fois par l'extrémité des diffuseurs, a ainsi créé dans la vase des "avant-trous" qui se sont agrandis lorsque les pompes de circulation ont été remises en service, permettant ainsi le dévasage des diffuseurs aval et de leur conduite de raccordement.

Un surcroît de précautions a été pris pour les ouvrages des tranches 3 et 4 . En particulier, les orifices des diffuseurs ont été fermés, avant même qu'ils soient immergés, par des obturations métalliques dont l'étanchéité est assurée par un joint gonflable.

\section{Mode d'exploitation des circuits}

Des précautions devront être prises pendant toute la durée d'exploitation de la Centrale. Elles se traduiront par les consignes suivantes destinées à éviter tout envasement ultérieur des ouvrages :

\subsection{Conduites d'amenée}

Si la disponibilité des pompes ne permet pas d'assurer le demi-débit dans une conduite, fermer les batardeaux de ses deux extrémités.

Cependant, la nécessité de maintenir en service le circuit secouru d'eau brute peut entraîner un envasement; dans ce cas, la technique de nettoyage consiste à mettre en communication les deux cheminées d'équilibre, à démarrer une pompe de la tranche dont la galerie est envasée et les deux pompes de l'autre tranche, de manière à aspirer en parallèle dans les deux galeries. Dans ces conditions, il s'établit dans la galerie envasée une vitesse de circulation supérieure à $1,5 \mathrm{~m} / \mathrm{s}$ qui permet l'érosion progressive des boues déposées. Cet essai a été réalisé et a donné toute satisfaction.

\subsection{Conduites de rejet}

En cas d'arrêt d'une ou plusieurs tranches, maintenir en service les pompes de circulation nécessaires pour que toutes les conduites de rejet soient parcourues par un débit au moins égal à la moitié de leur débit nominal.

Si la disponibilité des pompes ne permet pas d'appliquer cette consigne à une conduite, fermer le batardeau d'entrée de cette conduite et, si l'arrêt dépasse un mois, mettre en place les obturateurs sur les sorties de ses diffuseurs.

\subsection{Isolement de la station de pompage}

Pour lutter contre l'envasement provoqué par le marnage, des batardeaux à simple effet à joint gonflable seront mis en place, au droit du site, sur les galeries d'amenée et de rejet.

La mise en place de ces batardaux sur les galeries d'amenée est plus aisée que celle des doubles batardeaux situés en Gironde et peut être effectuée dans de meilleurs délais. Leur mise en place rapide permet également de s'affranchir du risque de voir se former une onde de boue au moment de la remise en suspension des vases déposées pendant l'arrêt. 


\section{Conclusion et enseignements}

La réalisation des ouvrages de prise et rejet d'eau de la Centrale du Blayais dans l'estuaire de la Gironde a soulevé des difficultés dues à la présence d'une quantité importante de vase. Ces difficultés se sont traduites à trois niveaux :

- intégration des contraintes d'exploitation dues à la vase dans la conception du projet,

- résolution de problèmes spécifiques apparus au cours de la réalisation, tels que le premier dévasage des conduites de rejet,

- définitions de consignes d'exploitation en fonction de la marche des tranches.

La solution de nombreux problèmes, fondée sur la connaissance de la vase, a permis de mieux appréhender le fonctionnement de certains mécanismes.

\section{Bibliographie}

[1] L.C.H.F. - Etude des propriétés physiques de la vase de Gironde Rapport L.C.H.F. C. Migniot, février 1970

[2] L.C.H.F. - Dynamique Sédimentaire Estuarienne Rapport L.C.H.F. C. Migniot, avril 1979.

[3] L.N.H. - Etude expérimentale de la dynamique des sédiments. fins. Rapport n ${ }^{\circ}$ 2. R. Bonnefille, septembre 1971.

[4] L.N.H. - Synthèse générale des données naturelles. Rapport $\mathrm{n}^{\circ}$ 21. R. Bonnefille, février 1972.
[5] L.N.H. - Etude des turbidités et des vitesses de chute de la vase en Gironde. R. Bonnefille. Allen, décembre 1973.

16) A.I.R.H. - Conception des rejets d'eau de refroidissement de la Centrale Electronucléaire du Blayais sur l'estuaire de la Gironde. J.D. Lepetit - M. Davesne, A.I.R.H. XIX Congrès 1981 .

[7] E.D.F. - Fonctionnement des pompes CRF fiche technique REP M 79.2. Vigneron.

[8] E.D.F. - Etude de la mise en service de purge CFF. E. REP W $900, n^{\circ} 220 \mathrm{M}$, Mathieu - Vigneron, janvier 1980.

19] I.G.B.A - Etude de la sédimentation encombrant la conduite de rejet de la centrale nucléaire de Braud et SaintLouis. P. Castaing.

- Etude d'une carotte de sédiments prélevés le 7/07/80, J.M. Jouanneau.

- Etude de la sédimentation encombrant les diffuseurs de la conduite de rejet, J,M. Jouanneau, 28 mai 1980.

[10] E.D.F. - Envasement des conduites d'eau de circulation, E. REP n ${ }^{\circ}$ 3, F. Lemelle, septembre 1979

[11] E.D.F. - Etude des conditions hydrauliques dans la conduite de rejet pendant les étapes de dévasage, $\mathrm{HL}-042$ 80 - 65, MM. Davesne - Kovacs, décembre 1980.

[12] E.D.F. - Centrale du Blayais, tranches 1-2. Dévasage de conduite d'eau de circulation. Note REP, G. Morlet, $11 \mathrm{sep}$ tembre 1980.

[13] E.D.F. - Région d'Equipement de Paris, Service TravauxEssais. Dévasage et mise en service des tuyauteries de rejet d'eau de circulation d'une paire de tranches. Procédure d'exécution d'essais C1 (BLOC1 PEE 30), Windels.

$114]$ E.D.F. - Dévasage des conduites de rejet - Comparaison des mesures in-situ avec les prévisions calculées, $\mathrm{HE}-042$ 81 - 37, M. Davesne, mai 1981.

[15] E.D.F. - Envasement dévasage et mise en service des tuyauteries d'amenée d'eau de circulation d'une paire de tranches, BL-001 Procédure d'exécution d'essai, Bloc 1 PEE 70, Lefèbre, août 1982.
M. le Président. - Je remercie M. LAURENT et $\mathrm{M}$. DAVESNE. $Y$ a-t-il des questions à poser sur cet intéressant problème?

M. GOGUEL. - Je voudrais dire mon intérêt pour votre exposé, qui pose le problème de l'envasement d'un long tuyau horizontal dans une eau turbide, certes, mais sans cummune mesure avec les concentrations de 400 à $500 \mathrm{~g} / \mathrm{litre}$ déposées.

J"ai eu l'occasion de voir en Algérie une conduite d'un diamètre de $1800 \mathrm{~m}$, d'un kilomètre de longueur, subhorizontale, qui était restée en attente quelques années entre une station de pompage et un canal. Cette conduite était envasée à plus de la moitié de sa section par une vase très compacte qu'il a fallu attaquer à la pioche, alors que l'eau, chargée certes, qui a pu pénétrer dans cette conduite, n'était jamais qu'à la densité de $1,05-1,10$

Autre exemple, une galerie de contrôle dans un grand barrage en construction, calée normalement juste au-dessus du niveau aval, le jour d'une petite crue est noyée par une eau de crue, done chargée à quelques dizaines de grammes par litre. Et, dans cette galerie de quelques centaines de mètres de longueur, 3 mètres de diamètre, il y a 2 mètres de vase qui doit partir à la pelle et à la pioche, alors qu'on a eu quelques mètres de charge seulement dessus.

Tout se passe comme si, après avoir déposé sa vase par décantation, l'eau claire s'en allait, était remplacée par une eau chargée à la même densité, et peu à peu, on arrive ainsi à remplir la conduite sur plusieurs mètres d'épaisseur, phénomène qui ne peut s'expliquer seulement par la décantation en place de l'eau qui a pu pénétrer à l'intérieur. Comme vous y faites allusion, il y a certainement eu des circulations.

M. DAVESNE. - Oui. Je suis tout à fait d'accord avec vous. D'ailleurs cela se produit également dans les ports. $\mathrm{Si}$, pour évaluer l'envasement d'une darse portuaire, on prend simplement la concentration moyenne de la vase en suspension, et qu'on suppose même que tout ce qui est apporté dans la darse pendant une demi-marée s'y dépose sans que rien n'en ressorte, on trouve des volumes d'envasement qui sont généralement sous-estimés d'un facteur 4 à 5 à la réalité. Il y a donc bien d'autres méca- 
nismes, qui mettent souvent en jeu des courants liés aux différences de densité venant, soit de la différence de salinité entre la darse et l'estuaire, soit éventuellement de la différence de densité entre l'eau chargée et l'eau moins chargée.

M. G. LEVY. - Y a-t-il d'autres centrales nucléaires dans d'autres estuaires qui donnent lieu à des problèmes du genre de celuj que vous avez traité pour le Blayais?

M. DAVESNE. - Dans le même estuaire, nous avons la Centrale d'Ambes, mais la prise d'eau est assez proche de la berge et le rejet s'effectue en canal de l'autre côté du bec. Il n'y a donc pas de tuyau. L'originalité du Blayais vient de ses 2 kilomètres de conduite ensouillée dans l'estuaire.
M. LAURENT. - Le seul autre endroit où il y a des canalisations est Paluel, mais il s'agit de galeries creusées dans le rocher et, de toutes façons, l'eau y est claire.

Les difficultés d'un estuaire comme la Gironde, par rapport aux problèmes qui peuvent se poser en Méditerranée par exemple viennent du fait qu'il n'y a aucune période dans l'année où on a de l'eau claire, ou si brièvement qu'on ne peut pas engager de travaux. D'autre part, la conduite des travaux ne peut se faire qu'aux étals de marées. Cette forte turbidité où on ne voit strictement rien pendant toute l'année, et le courant qui monte jusqu'à 1 à $1,5 \mathrm{~m} / \mathrm{s}$ rendent les moindres travaux extrêmement difficiles. 